\title{
A PROOF OF SCHWARTZ'S KERNEL THEOREM
}

\author{
H. GASK
}

\section{Introduction.}

We let $O_{x}$ and $O_{y}$ denote open sets in the Euclidean spaces $R^{n}$ and $R^{m}$ respectively and write $O_{x y}$ for the product $O_{x} \times O_{y}$. As usual, $\mathscr{D}\left(O_{x}\right)$, $\mathscr{D}\left(O_{y}\right)$ and $\mathscr{D}\left(O_{x y}\right)$ stand for the spaces of infinitely differentiable functions with compact supports (in $O_{x}, O_{y}$ and $O_{x y}$ respectively), equipped with the standard topology ([3]). The strong duals of these spaces will be denoted by $\mathscr{D}^{\prime}\left(O_{x}\right)$, etc.

We identify a locally integrable function $T$ in, e.g., $\mathscr{D}\left(O_{x}\right)$ with the distribution

$$
f \rightarrow\langle T, f\rangle=\int T(x) f(x) d x,
$$

and we shall use the integral as a notation for the value $\langle T, f\rangle$ of $T$ at $f$ also when $T$ is an arbitrary distribution.

Consider the space $\mathscr{A}$ of all separately continuous bilinear functionals $A$ on $\mathscr{D}\left(O_{x}\right) \times \mathscr{D}\left(O_{y}\right)$ with the topology of uniform convergence on products of bounded sets in $\mathscr{D}\left(O_{x}\right)$ and $\mathscr{D}\left(O_{y}\right)$. Any distribution $T$ in $\mathscr{D}^{\prime}\left(O_{x y}\right)$ gives rise to such a functional $A$ by specialisation to products of functions of $x$ and $y$ :

$$
(\Lambda T)(f, g)=\langle T, f(x) g(y)\rangle=A(f, g) .
$$

The kernel theorem says that the mapping

$$
T \rightarrow \Lambda T=A
$$

is a linear homeomorphism between $\mathscr{D}^{\prime}\left(O_{x y}\right)$ and $\mathscr{A}$. In particular, there exists to any $A$ in $\mathscr{A}$ precisely one "kernel" $T$ in $\mathscr{D}^{\prime}\left(O_{x y}\right)$ such that

$$
A(f, g)=\int T(x, y) f(x) g(y) d x d y \text {. }
$$

The theorem was first proved by Schwartz [4], and a much simplified proof has then been given by Ehrenpreis [2]. Our proof is close to that of Ehrenpreis but has the advantage that we can easily show that $\Lambda$ in (1) is one-to-one and onto $\mathscr{A}$, before proving that it is a topological isomorphism. The proof of the former half of the theorem can thus be

Received May 20, 1960. 
made independent of most of the topological concepts necessary for the latter. A by-product of the proof is also a simple estimate of the local order of $T$ in terms of the local order of $A$.

The theorem remains true if, e.g., $\mathscr{D}$ is replaced by the space $\mathscr{E}$ of infinitely differentiable functions, or if $O_{x}=R^{n}, O_{y}=R^{m}$ and $\mathscr{D}$ is replaced by the space $\mathscr{S}$ of Schwartz (see [3]). The proof given here is easily adapted to these cases.

In what follows, $U$ and $V$ will always denote compact sets in $O_{x}$ and $O_{y}$ respectively. By $\mathscr{D}(U)$ we shall denote the subspace of $\mathscr{D}\left(O_{x}\right)$ whose elements have their supports in $U$, and analogously for $\mathscr{D}(V)$ and $\mathscr{D}(U \times V)$.

\section{Some estimates for Fourier series.}

To any given $U$ and $V$ we can find a number $a$, such that $U$ and $V$ are contained in cubes with sides $2 a-2$, say, in $R^{n}$ and $R^{m}$. We let $K_{x}$ and $K_{y}$ stand for the corresponding cubes with side $2 a$ and write

and

$$
e_{p}(x)=\gamma_{n} \cdot \exp \left\{i \pi a^{-1}\left(p_{1} x_{1}+p_{2} x_{2}+\ldots+p_{n} x_{n}\right)\right\}
$$

$$
e_{q}(y)=\gamma_{m} \cdot \exp \left\{i \pi a^{-1}\left(q_{1} y_{1}+q_{2} y_{2}+\ldots+q_{m} y_{m}\right)\right\} \text {. }
$$

Here $p$ and $q$ are $n$ - and $m$-tuples of integers and the constants $\gamma_{n}$ and $\gamma_{m}$ are chosen such that the functions are orthonormal. Any function $h(x, y)$ in $\mathscr{D}(U \times V)$ can be expanded in a Fourier series in $K_{x} \times K_{y}$ :

$$
h(x, y)=\sum a_{p q} e_{p}(x) e_{q}(y),
$$

the sum taken over all $p$ and $q$. The coefficients in (2) are given by wellknown integral formulas, and integrations by parts in these formulas yield the classical estimates

$$
\left|a_{p q}\right| \leqq C_{j}|h|_{j}(1+|p|+|q|)^{-j} .
$$

Here $C_{j}$ is a constant independent of $h, j$ is an arbitrary positive integer, $|p|$ and $|q|$ are defined as $\Sigma\left|p_{v}\right|$ and $\Sigma\left|q_{v}\right|$ respectively and $|h|_{j}$ stands for $\sup _{x} \Sigma_{|\alpha| \leqq j}\left|D^{\alpha} h(x, y)\right|$. If $\varphi(x)$ and $\psi(y)$ are in $\mathscr{D}\left(K_{x}\right)$ and $\mathscr{D}\left(K_{y}\right)$ and identically one on $U$ and $V$, the expansion

$$
h(x, y)=\sum a_{p q} \varphi(x) e_{p}(x) \psi(y) e_{q}(y)
$$

holds in the whole of $O_{x} \times O_{y}$. We shall write (4) in the form

$$
h(x, y)=\sum \lambda_{p q} f_{p}(x) g_{q}(y)
$$

with $f_{p}(x)$ and $g_{q}(y)$ proportional to $\varphi(x) e_{p}(x)$ and $\psi(y) e_{q}(y)$ : 


$$
\begin{aligned}
& \alpha_{p q} f_{p}(x)=\varphi(x) e_{p}(x), \\
& \beta_{p q} g_{q}(y)=\psi(y) e_{q}(y) .
\end{aligned}
$$

With these notations the coefficients in (5) are given by

$$
\lambda_{p q}=\alpha_{p q} \beta_{p q} a_{p q} .
$$

The proportionality factors shall be chosen in a suitable way, expressed in the following lemmas.

Lemma 1. Let $h$ be a function in $\mathscr{D}(U \times V)$ and $k$ and $l$ given positive integers. Then $f_{p}$ and $g_{q}$ in (5) can be chosen such that

and

$$
\left|f_{p}\right|_{k} \leqq 1
$$

for all $p$ and $q$ and

$$
\left|g_{q}\right|_{l} \leqq 1
$$

$$
\sum\left|\lambda_{p q}\right| \leqq C|h|_{k+l+n+m+2}
$$

with a constant $C$ independent of $h$.

Proof. We write (4) as

$h(x, y)=\sum a_{p q}(1+|p|)^{k}(1+|q|)^{l}\left[(1+|p|)^{-k} \varphi(x) e_{p}(x)\right]\left[(1+|q|)^{-l} \psi(y) e_{q}(y)\right]$, and choose the functions in square brackets for $f_{p}$ and $g_{q}$. The estimates (3) and some straight-forward calculations then give the lemma.

LEMma 2 (Ehrenpreis). Let $\left\{a_{v}\right\}_{1}^{\infty}$ be a sequence of positive real numbers and $h$ any function in $\mathscr{D}(U \times V)$ satisfying

$$
|h|_{\nu} \leqq a_{\nu}, \quad v=1,2, \ldots
$$

Then there exists another sequence $\left\{b_{\nu}\right\}_{1}^{\infty}$, depending only on the original one and not on $h$, such that with a suitable choice of $f_{p}$ and $g_{q}$ in (5) we have

$$
\left|f_{p}\right|_{v} \leqq b_{v}, \quad\left|g_{q}\right|_{v} \leqq b_{v}, \quad v=1,2, \ldots,
$$

for all $p$ and $q$, and also

$$
\sum\left|\lambda_{p q}\right| \leqq 1
$$

Proof. The lemma expresses the fact that if $h$ is in some bounded set in $\mathscr{D}(U \times V)$, the expansion (5) can be made such that (6) holds with $f_{p}$ and $g_{q}$ in fixed bounded sets in $\mathscr{D}(U)$ and $\mathscr{D}(V)$ respectively. For the proof, put

and hence

$$
\begin{aligned}
f_{p}(x) & =\left|a_{p q}\right|^{\frac{1}{4}} \varphi(x) e_{p}(x), \\
g_{q}(y) & =\left|a_{p q}\right|^{\frac{1}{4}} \psi(y) e_{q}(y) \\
\lambda_{p q} & =a_{p q}\left|a_{p q}\right|^{-\frac{1}{2}}
\end{aligned}
$$


in (4). Then the rapid decrease of the coefficients $a_{p q}$, expressed by (3), is easily seen to give the lemma.

\section{The kernel theorem.}

As stated above, $\mathscr{A}$ denotes the set of all separately continuous bilinear functionals on $\mathscr{D}\left(O_{x}\right) \times \mathscr{D}\left(O_{y}\right)$, and in what follows we shall simply write $\mathscr{T}$ for $\mathscr{D}^{\prime}\left(O_{x y}\right)$.

It is a classical fact that the restriction to $\mathscr{D}(U) \times \mathscr{D}(V)$ of any $A \in \mathscr{A}$ is continuous (see, e.g., $[1$, p. 83]) and so there exist a constant $C$ and integers $k$ and $l$ (depending, of course, on $A$ ) for which

$$
|A(f, g)| \leqq C|f|_{k}|g|_{l}, \quad f \in \mathscr{D}(U), \quad g \in \mathscr{D}(V) .
$$

As stated in the introduction, there is a mapping $\Lambda$ of $\mathscr{T}$ into $\mathscr{A}$, defined by (1). We shall now first prove that the range of this mapping is the whole of $\mathscr{A}$ and that it is one-to-one.

THEOREM 1. For any separately continuous functional $A$ on $\mathscr{D}\left(O_{x}\right) \times \mathscr{D}\left(O_{y}\right)$ there exists precisely one distribution $T$ in $\mathscr{T}$ such that

$$
(\Lambda T)(f, g)=\langle T, f(x) g(y)\rangle=A(f, g)
$$

for all $(f, g)$ in $\mathscr{D}\left(O_{x}\right) \times \mathscr{D}\left(O_{y}\right)$.

Proof. We begin by restricting $A$ to $\mathscr{D}(U) \times \mathscr{D}(V), U$ and $V$ compact, and write an arbitrary $h$ in $\mathscr{D}(U \times V)$ in the form given by lemma 1. If $k$ and $l$ are integers such that (7) holds for our given $A$ we find

$$
\sum\left|\lambda_{p q}\right|\left|A\left(f_{p}, g_{q}\right)\right| \leqq C|\hbar|_{k+l+n+m+2}
$$

with $C$ independent of $h$. We define $T$ by

$$
\langle T, h\rangle=\sum \lambda_{p q} A\left(f_{p}, g_{q}\right)
$$

and conclude from (9) that $T$ is a distribution on $U \times V$ of order $\leqq k+l+n+m+2$. It is clear that (8) holds for this $T$ and also that $T$ is uniquely determined by $A$, for if $A$ vanishes we infer from (10) that $T(h)=0$ on all finite sums $h=\sum \lambda_{p q} f_{p} g_{q}$, and as the set of such sums is total in $\mathscr{D}(U \times V)$ the distribution $T$ must vanish.

Now, $O_{x}$ and $O_{y}$ are unions of compact sets in each of which the existence of a unique $T$ has been proved, and the theorem follows.

Theorem 2. The mapping $\Lambda$ defined by (8) is a linear homeomorphism.

Proof. The topologies on $\mathscr{A}$ and $\mathscr{T}$ as introduced above are defined by the seminorms 


$$
\begin{aligned}
\varrho_{B_{x} B_{y}}(A) & =\sup |A(f, g)|, & & f \in B_{x}, \quad g \in B_{y}, \\
\sigma_{B_{x y}}(T) & =\sup |\langle T, h\rangle|, & & h \in B_{x y},
\end{aligned}
$$

where $B_{x}, B_{y}$ and $B_{x y}$ are bounded sets in $\mathscr{D}\left(O_{x}\right), \mathscr{D}\left(O_{y}\right)$ and $\mathscr{D}\left(O_{x y}\right)$ respectively.

It is clear that $\Lambda$ is linear. In order that $\Lambda$ be a homeomorphism it is necessary and sufficient that it is bicontinuous, i.e. that $\Lambda$ and $\Lambda^{-1}$ are both continuous.

Let $\varrho_{B_{x} B_{y}}$ be an arbitrary seminorm on $\mathscr{A}$. Then

$$
\varrho_{B_{x} B_{y}}(\Lambda T)=\varrho_{B_{x} B_{y}}(A)=\sup _{f \in B_{x}, g \in B_{y}}|A(f, g)|=\sup _{f \in B_{x}, g \in B_{y}}|\langle T, f g\rangle| .
$$

It is easy to see that for any bounded sets $B_{x} \subset \mathscr{D}\left(O_{x}\right)$ and $B_{y} \subset \mathscr{D}\left(O_{y}\right)$ there exists a bounded set $B_{x y} \subset \mathscr{D}\left(O_{x y}\right)$ such that all products $f g$ are in $B_{x y}$ whenever $f$ is in $B_{x}$ and $g$ in $B_{y}$. Hence

$$
\sup _{f \in B_{x}, g \in B_{y}}|\langle T, f g\rangle| \leqq \sup _{h \in B_{x y}}|\langle T, h\rangle|=\sigma_{B_{x y}}(T),
$$

and so $\Lambda$ is continuous. Conversely, if $\sigma_{B_{x y}}$ is a seminorm on $\mathscr{T}$ we find

$$
\sigma_{B_{x y}}\left(\Lambda^{-1} A\right)=\sigma_{B_{x y}}(T)=\sup _{h \in B_{x y}}|\langle T, h\rangle|=\sup _{h \in B_{x y}}\left|\sum \lambda_{p q} A\left(f_{p}, g_{q}\right)\right|,
$$

where $h$ has been expanded as in lemma 2, and thus

$$
\sup _{h \in B_{x y}}\left|A\left(f_{p}, g_{q}\right)\right| \leqq \sup _{f \in B_{x}, g \in B_{y}}|A(f, g)|=\varrho_{B_{x} B_{y}}(A),
$$

if $B_{x}$ and $B_{y}$ are those bounded sets in $\mathscr{D}\left(O_{x}\right)$ and $\mathscr{D}\left(O_{y}\right)$ which contain all $f_{p}$ and $g_{q}$ according to lemma 2. From (6) we now conclude

$$
\sigma_{B_{x y}}\left(\Lambda^{-1} A\right) \leqq \sup _{h \in B_{x y}}\left|A\left(f_{p}, g_{q}\right)\right| \sum\left|\lambda_{p q}\right| \leqq \varrho_{B_{x} B_{y}}(A),
$$

and thus $\Lambda^{-1}$ is also continuous and the theorem is proved.

Remark. For a given $A$ in $\mathscr{A}$ we can define a functional $L$ on $\mathscr{D}\left(O_{y}\right)$ by

$$
\langle L, g\rangle=A(f, g), \quad f \in \mathscr{D}\left(O_{x}\right), \quad g \in \mathscr{D}\left(O_{y}\right),
$$

and $L$ is immediately seen to be a distribution in $\mathscr{D}^{\prime}\left(O_{y}\right)$ for every $f$. Hence every $A$ in $\mathscr{A}$ gives rise to a mapping $\Gamma$ from $\mathscr{D}\left(O_{x}\right)$ to $\mathscr{D}^{\prime}\left(O_{y}\right)$ :

$$
(\Gamma f)(g)=A(f, g),
$$

and it is easily checked that this mapping is linear and continuous. Conversely, via formula (11) every such linear continuous mapping is seen to define a bilinear functional in $\mathscr{A}$. 
The usual strong topology on the space $\mathscr{L}$ of all continuous linear mappings from $\mathscr{D}\left(O_{x}\right)$ to $\mathscr{D}^{\prime}\left(O_{y}\right)$ is defined by the seminorms

$$
\theta(\Gamma)=\sup _{f \in B_{x}} \tau(\Gamma f)
$$

where $\tau$ is a seminorm on $\mathscr{D}^{\prime}\left(O_{y}\right)$ and $B_{x}$ a bounded set in $\mathscr{D}\left(O_{x}\right)$. Therefore

$$
\theta(\Gamma)=\sup _{f \in B_{x}} \tau(\Gamma f)=\sup _{f \in B_{x}, g \in B_{y}}|\langle\Gamma f, g\rangle|=\sup _{f \in B_{x}, g \in B_{y}}|A(f, g)|=\varrho_{B_{x} B_{y}}(A) .
$$

Thus the kernel theorem states that the spaces $\mathscr{T}$ and $\mathscr{L}$ are homeomorphic, and this formulation of the theorem was the one used in the previous proofs.

\section{REFERENCES}

1. N. Bourbaki, Topologie générale, Ch. IX (Act. Sci. Ind. 1045), Paris, 1958.

2. L. Ehrenpreis, On the theorem of kernels of Schwartz, Proc. Am. Math. Soc. 7 (1956), 713-718.

3. L. Schwartz, Théorie des distributions I and II, Paris, 1950 and 1951.

4. L. Schwartz, Espaces de fonctions differentiables à valeurs vectorielles, J. Analyse Math. 4 (1954-55), 88-148.

UNIVERSITY OF LUND, SWEDEN 